\title{
Diversity and shared T-cell receptor repertoire analysis in esophageal squamous cell carcinoma
}

\author{
TOMOYA SUDO ${ }^{1}$, AKIHIKO KAWAHARA $^{2}$, KAZUO ISHI $^{3}$, ATSUSHI MIZOGUCHI $^{4}$, SACHIKO NAGASU $^{1}$, \\ MASASHI NAKAGAWA ${ }^{1}$, MASAHIRO FUJISAKI ${ }^{1}$, HARUHIRO HINO $^{1}$, KOUHEI SAISHO $^{1}$, HIDEAKI KAKU $^{1}$, \\ SATORU MATONO $^{1}$, NAOKI MORI ${ }^{1}$, JUN AKIBA ${ }^{2}$, AKIRA YAMADA $^{5}$ and YOSHITO AKAGI $^{1}$ \\ Departments of ${ }^{1}$ Surgery and ${ }^{2}$ Hospital Diagnosis; ${ }^{3}$ Biostatistics Center; ${ }^{4}$ Department of Immunology, \\ Kurume University School of Medicine; ${ }^{5}$ Cancer Vaccine Development Division, Research for Innovative Cancer Therapy, \\ Kurume University School of Medicine, Kurume, Fukuoka 830-0011, Japan
}

Received January 18, 2021; Accepted May 24, 2021

DOI: 10.3892/ol.2021.12879

\begin{abstract}
The tumor immune response is dependent on the interaction between tumor cells and the T-cell subset expressing the T-cell receptor (TCR) repertoire that infiltrates into the tumor microenvironment. The present study explored the diversity and shared TCR repertoires expressed on the surface of locoregional $\mathrm{T}$ cells and identified the T lymphocyte subsets infiltrating into esophageal squamous cell carcinoma (ESCC), in order to provide insight into the efficiency of immunotherapy and the development of a novel immune-oriented therapeutic strategy. A total of 53 patients with ESCC were enrolled in the present study, and immunohistochemical analysis of CD3, CD8, CD45RO, FOXP3, CD274, HLA class I and AE1/AE3 was performed. Digital pathological assessment was performed to evaluate the expression level of each marker. The clinicopathological significance of the immuno relation high (IR-Hi) group was assessed. Adaptor ligation PCR and next-generation sequencing were performed to explore the diversity of the TCR repertoire and to investigate the shared TCR repertoire in the IR-Hi group. Repertoire dissimilarity index (RDI) analysis was performed to assess the diversity of TCR, and the existence of shared TCR $\alpha$ and TCR $\beta$ was also investigated. Further stratification was performed according to the expression of markers of different T-cell subsets. Patients were stratified into IR-Hi and immuno relation low (IR-Lo) groups. Cancer-specific survival and recurrence-free survival rates were significantly improved in the IR-Hi group compared with in the IT-Lo group. The diversity of the TCR repertoire was significantly higher in the IR-Hi group. TCR repertoire analysis revealed 27 combinations of TCR $\alpha$ and 23
\end{abstract}

Correspondence to: Dr Tomoya Sudo, Department of Surgery, Kurume University School of Medicine, 67 Asahi-machi, Kurume, Fukuoka 830-0011, Japan

E-mail: sudou_tomoya@med.kurume-u.ac.jp

Key words: comprehensive T-cell receptor repertoire analysis, esophageal squamous cell carcinoma combinations of TCR $\beta \mathrm{VJ}$ regions that were shared among the IR-Hi group. The IR-Hi group was divided into three clusters. Overall, the current findings revealed that the IR-Hi group maintained the diversity of TCR, and a portion of the IR-Hi cases held the T cells with shared TCR repertoires, implying recognition of shared antigens. The prognosis of patients with ESCC was affected by the existence of immune response cells and may possibly be stratified by the T-cell subsets.

\section{Introduction}

In 2018, esophageal cancer was the seventh leading cause of cancer-associated mortality and the sixth leading cause of morbidity worldwide (1). In particular, patients in East Asian regions, including Japan, have esophageal squamous cell carcinoma (ESCC), which is the most common histopathological subtype in the region (2). The major tumor depth of ESCC is within the subserosa of the esophagus; however, even in the shallower depth, 30-50\% of cases are identified with lymph node metastasis (3). Therefore, numerous patients are diagnosed at an advanced stage, and it can be difficult to treat the disease with monotherapy. Accordingly, the standard treatment for ESCC is esophagectomy combined with three-field lymphadenectomy and neoadjuvant or adjuvant chemoradiation therapy $(4,5)$. Moreover, the immune checkpoint inhibitor pembrolizumab has been approved as a second-line treatment option by the United States Food and Drug Administration (6,7); this therapeutic strategy is expected to be widely applied.

It has been previously reported that patients with ESCC with a high percentage of tumor-infiltrating lymphocytes (TILs) exhibit significantly improved prognoses compared with patients with a low percentage of TILs (8). Similar findings have been reported in other gastrointestinal cancers (9-13), suggesting that the existence of immune cells, particularly lymphocytes, in the tumor region is important for fighting the disease. TILs include components of the T-cell subsets including cytotoxic $\mathrm{T}$ cells, memory $\mathrm{T}$ cells, helper $\mathrm{T}$ cells and regulatory $\mathrm{T}$ cells (14-16). The TIL component balance determines the immunologic anti- or pro-tumor effect (17). Therefore, analysis of the patterns of TIL subsets may reveal the most appropriate antitumor effect. 
Furthermore, T-cell receptors (TCRs) on the surface of $\mathrm{T}$ cells specifically recognize the antigens presented on major histocompatibility complex molecules on tumor cells. TCRs are composed of $\alpha$ and $\beta$ chains. Both chains have variable (V) and joining ( $\mathrm{J}$ ) regions that depend on the TCR $\alpha$ and TCR $\beta$ DNA rearrangements during T-cell maturation, and function to maintain TCR diversity to allow specific TCR recognition of various pathogens invading into the body or of harmful malfunctioning cells (18).

The tumor tissue is an aggregation of heterogeneous tumor cells; therefore, the existence of $\mathrm{T}$ cells expressing diverse TCRs is essential for effective antitumor immune responses (19-21). Additionally, among the diverse TCRs, identification of tumor-specific shared TCR sequences, including TCR gene rearrangements, may facilitate the development of novel immune therapies, such as chimeric antigen receptor $\mathrm{T}$ (CAR-T) cell therapy (22).

Previously, comprehensive TCR sequence analysis was laborious due to the huge TCR diversity; however, next-generation sequencing (NGS) technologies now enable the investigation of the numerous TCR repertoires. Accordingly, the primary objective of the present study was to investigate the diversity of the TCR repertoire and explore the shared TCR repertoire on $\mathrm{T}$ cells within the tumor environment of patients with ESCC, using a combination of adaptor ligation PCR and NGS. The secondary objective was to assess the impact of the T-cell subset pattern on the immuno relation (IR) group in patients with ESCC.

\section{Materials and methods}

Patients and institutional review board approval. In total, 124 patients with ESCC underwent esophagectomy at the Department of Surgery of Kurume University Hospital (Kurume, Japan) between April 2013 and March 2017. Among these patients, the cases with neoadjuvant chemotherapy and a lack of clinical information cases were excluded from the study, and a total of 53 cases were enrolled in the present study. An overview of the patients' characteristics, pathological staging and clinicopathological factors is shown in Table SI. All patients underwent subtotal esophagectomy, including three-field lymphadenectomy. Additional adjuvant chemotherapy was performed after surgery in patients with pathological lymph node metastasis. Patients were regularly checked every 3 months in the first year and following every 6 months for 5 years after surgery. The mean observation period after surgery was $565 \pm 525.54$ days. TNM classification (8th edition) was used for clinical and pathological staging (23). Written informed consent was obtained from all enrolled patients. The patient selection process, clinicopathological information obtained during analysis and experimental protocol of the present study complied with the guidelines approved by the Ethics Committee of Kurume University School of Medicine (approval no. 282).

Immunohistochemical (IHC) staining. Formalin-fixed ( $10 \%$ at room temperature for $48 \mathrm{~h}$ ) paraffin-embedded tissue samples were sliced to a thickness of $4 \mu \mathrm{m}$ and examined on coated glass slides. The tissues were deparaffinized and labeled with antibodies using a BenchMark ULTRA (Ventana Medical Systems, Inc.) and Bond-Max autostainer (Leica
Microsystems, Inc.). Each slide was heat-treated using CC1 retrieval solution (Ventana Medical Systems, Inc.) at $99^{\circ} \mathrm{C}$ for $60 \mathrm{~min}$ and incubated with the primary antibody at room temperature for $30 \mathrm{~min}$, followed by incubation with a streptavidin-biotin complex using 3,3'-diaminobenzidine (UltraVIEW DAB detection kit; Ventana Medical Systems, Inc.) as the chromogen. The following primary antibodies were used: Anti-CD3 (1:300; clone LN10; cat. no. CD3-565-L-CE; Leica Microsystems, Inc.), anti-CD8 (1:200; clone 4B11; cat. no. CD8-4B11-L_CE; Leica Microsystems, Inc.), anti-CD45RO (1:5,000; clone UCLH1; cat. no. Ab23; Abcam), anti-FOXP3 (1:100; Abcam), anti-CD274/programmed cell death 1 ligand 1 (PD-L1; 1:100; clone E1L3N; cat. no. 13684; Cell Signaling Technology, Inc.), anti-human leukocyte antigen (HLA) class I (1:1,000; cat. no. ab52922; Abcam) and anti-cytokeratin (AE1/AE3; 1:400; cat. no. GA05361-2; Dako; Agilent Technologies, Inc.).

IHC evaluation of $C D 3, C D 8, C D 45 R O, F O X P 3, C D 274$, $H L A$ class $I$ and AE1/AE3. A digital pathology procedure was used to evaluate the prepared slides to avoid the subjectivity of evaluation by pathologists. All of the routinely stained hematoxylin and eosin (H\&E) slides- and IHC-stained slides were captured by the NanoZoomer XR digital scanner (Hamamatsu Photonics KK), and digital data were acquired. For analysis of CD3, CD8, FOXP3 and CD45RO, five positions within the center of the tumor (CT) and along the invasive margin (IM) of the tumor were selected, and images at x20 magnification were captured and digitized (Fig. S1). For CD274, HLA class I and AE1/AE3, images at x1.25 magnification were captured and processed using ImageJ v1.41 (National Institutes of Health) image-processing software (24). Briefly, a color deconvolution procedure was performed on the original images, red-colored images were selected, and binary images were generated. For binary images of CD3, CD8, FOXP3 and CD45RO, five positions were selected, within the CT and IM of the tumor, and the numbers of dots representing $\mathrm{T}$ cells were counted. The median staining intensity values at the CT and IM were calculated. For binary images of CD274, HLA class I and AE1/AE3, the tumor area was calculated, and the expression of CD274 was standardized by dividing the value by that of AE1/AE3. Similarly, the expression of HLA class I on the tumor was obtained using the same procedure (Figs. S2 and S3).

Positive staining for CD3, CD8, FOXP3 and CD45RO was limited in lymphocytes. Digital evaluation of these markers was performed by counting the positive dots. Positive evaluation of CD274, HLA class I and AE1/AE3 in a tumor was complex since positive staining was observed in tumor cells and adjacent surrounding structures. Therefore, the value of positive area of each marker was calculated, and this value was standardized by the area of AE1/AE3 representing epithelial cells.

Identification for IR groups and further subset stratification. To elucidate the involvement of lymphocytes and relevant molecules in ESCC, the expression values calculated with ImageJ for CD3, CD8, CD45RO, FOXP3, CD274 and HLA were utilized. Principal component analysis (PCA) $(25,26)$ was performed using the K-means clustering method and the entered cases were divided into two groups. The group showing the same vector 
pattern of all the markers through the PCA was defined as IR high (IR-Hi) group $(n=21)$ and the other group showing no such trend was defined as IR low (IR-Lo) group $(n=32)$.

Hierarchical clustering analysis with the Ward method was conducted to exploratively visualize the level of T-cell subset involvement in the IR-Hi group. The analysis was performed using JMP v13.0 software (SAS Institute, Inc.).

RNA extraction. Small sections of tumor tissues and corresponding normal tissues were collected and stored in RNAlater reagent (Thermo Fisher Scientific, Inc.) at $-80^{\circ} \mathrm{C}$ until use. mRNA was extracted from the tissue sections using an AllPrep kit (Qiagen $\mathrm{GmbH}$ ) according to the manufacturer's protocol. The extracted mRNA samples were stored at $-80^{\circ} \mathrm{C}$ until use for TCR analysis.

Unbiased amplification of TCR genes and NGS. Among the 53 cases included in the cluster analysis, the stored mRNA samples five cases from each of the IR-Hi and IR-Lo groups were randomly selected for unbiased TCR amplification by reverse transcription (RT)-PCR followed by NGS. An NGS technology for unbiased TCR repertoire analysis developed by Repertoire Genesis Inc. was used. Briefly, unbiased adaptor ligation RT-PCR was performed as described previously (27). Total RNA was reverse transcribed into cDNA using Superscript III reverse transcriptase (Invitrogen; Thermo Fisher Scientific, Inc.) and the BSL-18E primer containing poly $_{18}$ as previously reported (27) and a NotI site. The second strand of the cDNA was synthesized using Escherichia coli DNA polymerase I (Invitrogen; Thermo Fisher Scientific, Inc.), E. coli DNA ligase (Invitrogen; Thermo Fisher Scientific, Inc.) and RNase H (Invitrogen; Thermo Fisher Scientific, Inc.). The double-stranded cDNA fragments were blunt-ended using T4 DNA polymerase (Invitrogen; Thermo Fisher Scientific, Inc.). The P10EA/P20EA adapter was ligated to the 5' end of the double-stranded cDNA and then digested with the Not I restriction enzyme at $37^{\circ} \mathrm{C}$ for $2 \mathrm{~h}$. After removal of adaptor and primer sequences using a MinElute Reaction Cleanup kit (Qiagen $\mathrm{GmbH}$ ), according to the manufacturer's instructions, PCR was performed using KAPA HiFi DNA Polymerase (Kapa Biosystems; Roche Diagnostics) and a primer specific to either the TCR $\alpha$-chain constant region (CA1) or $\beta$-chain constant region (CB1), and a primer specific to P20EA (27). The primer sequences used in the PCR method are available in Table SII (28). The PCR conditions were as follows: 20 cycles of $98^{\circ} \mathrm{C}$ for $20 \mathrm{sec}, 65^{\circ} \mathrm{C}$ for $30 \mathrm{sec}$ and $72^{\circ} \mathrm{C}$ for $1 \mathrm{~min}$. The second PCR was performed using either the CA2 or CB2 primers and the P20EA primer under the same PCR conditions. Amplicons were prepared by amplification of the second PCR products using P20EA-ST1 and either CA-ST1 or CB-ST1 (27). After PCR amplification, index (barcode) sequences were added using the Nextera XT Index kit v2, Set A (Illumina, Inc.). The indexed amplicon products were mixed at equimolar concentrations and quantified using the Qubit 2.0 Fluorometer (Thermo Fisher Scientific, Inc.). Sequencing was performed using the Illumina MiSeq paired-end platform (2x300 bp).

TCR repertoire analyses. All the paired-end reads were classified based on index sequences. Assignment of sequences was performed by determining sequences with the highest identity in a dataset of reference sequences from the international ImMunoGeneTics information system ${ }^{\circledR}$ (IMGT) database (http://www.imgt.org). Data processing, assignment and data aggregation were automatically performed using repertoire analysis software originally developed by Repertoire Genesis (RG) Inc.; the RG software ver.1.0 is composed of sequence homology searches using BLATN, an automatic aggregation program, a graphics program for TCR variant (TRV) and TCR Joining (TRJ) usage, and CDR3 length distribution. Sequence identities at the nucleotide level between query and entry sequences were automatically calculated. Parameters that increased sensitivity and accuracy (E-value threshold, minimum kernel and high-scoring segment pair score) were carefully optimized for respective repertoire analysis. Nucleotide sequences of CDR3 regions, ranging from the conserved cysteine at position 104 (Cys104) of IMGT nomenclature to the conserved phenylalanine at position 118 (Phe118) and the following glycine (Gly119), were translated to deduce the amino acid sequences. A unique sequence read (USR) was defined as a sequence read having no identity in TRV or TRJ and in the deduced amino acid sequence of CDR3 with the other sequence reads. The copy numbers of identical USRs were automatically counted by RG software in each sample and then ranked in order of the copy number. Percentage occurrence frequencies of sequence reads with TCR $\alpha$ variable (TRAV), TCR $\alpha$ joining (TRAJ), TCR $\beta$ variable (TRBV) and TCR $\beta$ joining (TRBJ) genes in total sequence reads were calculated.

Statistical analysis and evaluation of the TCR repertoire dissimilarity in each case. The acquired NGS data were processed for a complete comprehensive analysis of the TCR repertoire. The combinations of the TRAV and TRAJ regions and the TRBV and TRBJ regions that were significantly $(\mathrm{P}<0.05)$ and commonly elevated in the IR-Hi group were selected as the shared combination of the TRAVITRAJ and TRBVITRBJ repertoire.

Fisher's exact test was performed using the Bioconductor package edgeR in R (v3.4.2) (Foundation for Statistical Computing, https://www.r-project.org/foundation) $(29,30)$ to retrieve these analyses. To further ensure the significance of the shared combination of TRAVITRAJ and TRBVITRBJ repertoire, Mann Whitney $U$ tests were simultaneously conducted and the combinations with $\mathrm{P}<0.05$ in both tests were selected as statistically significant.

Dissimilarity analysis of each case (cases 1-10) was conducted using the average repertoire dissimilarity index (RDI) analysis $(31,32)$. The average RDI was calculated using the following procedure. The singleton repertoires were retracted from the TCR repertoire combinations data identified as statistically significant in previous analyses and 500 repertoire combinations were randomly collected using the bootstrap restoration extractive maneuver. The frequency of each repertoire was calculated using the calcVDJcounts function in the RDI package (https://rdocumentation.org/packages/ rdi/versions/1.0.0) (ver.1.0.0) of R (ver.3.6.1). The RDIs were acquired for each case using the calcRDI() function, and the average values of the RDIs were calculated. Cases in which RDI was difficult to calculate owing to the low frequency 
A

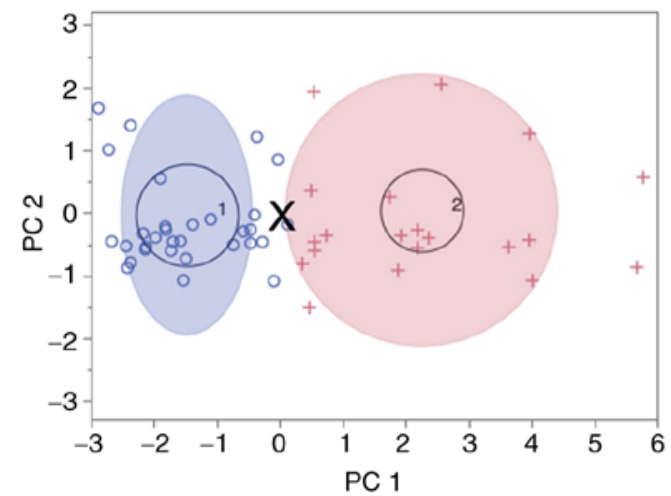

B

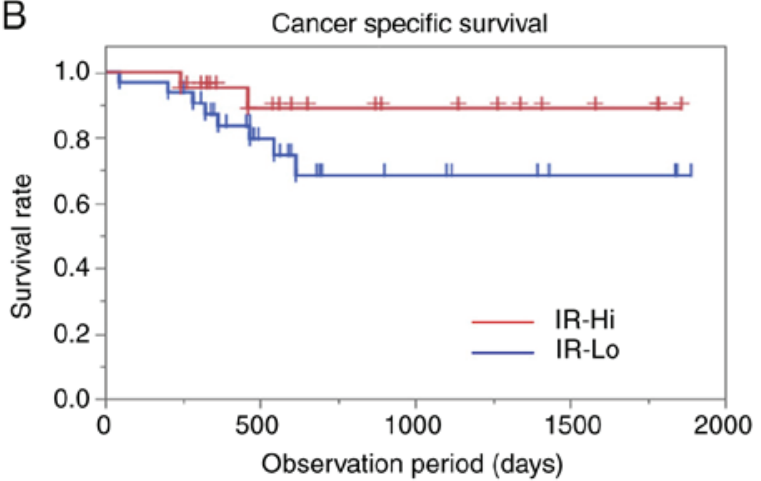

Log-rank $P=0.0487$

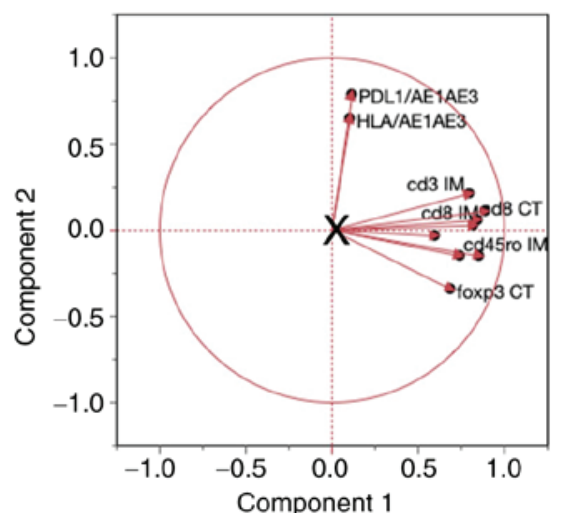

C

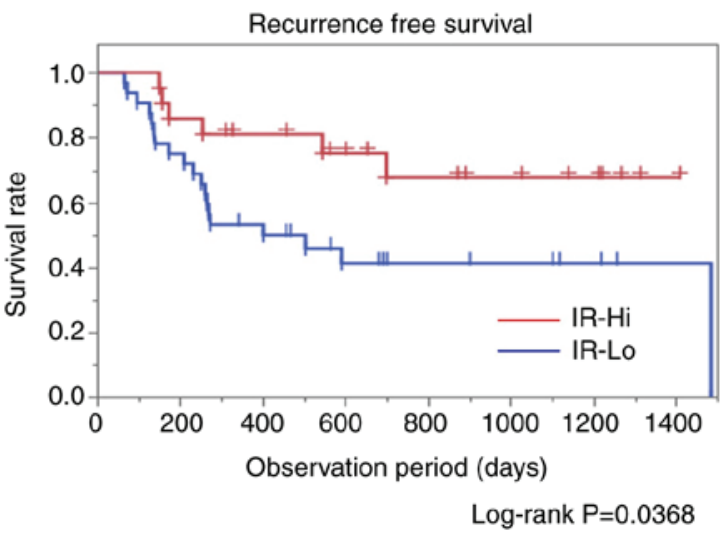

Figure 1. Identification for IR groups using PCA with K-means method and prognostic comparison using the log-rank test. (A) Overview of the PCA with K-means clustering method (left). Each blue circle and red cross marks indicate classified cases into cluster 1 (IR-Lo) and cluster 2 (IR-Hi). The blue and red ellipses indicate $95 \%$ confidence areas. The ' $\mathrm{X}$ ' mark in the middle of the figure indicates a specific point to divide the cases into two clusters. Correlative direction diagram in each parameter used in the calculation by PCA (right). Introduced values to determine PC1 and PC 2 were calculated using the values for the CT and IM for CD3, CD8, CD45RO, FOXP3, CD274 and HLA. The length and direction of each line indicate the strength and the correlation similarity between each parameter. (B) Cancer-specific survival and (C) recurrence-free survival comparison between the IR-Hi and IR-Lo groups. Both survival curves were compared using log-rank tests, and results with $\mathrm{P}<0.05$ were considered statistically significant. + and I indicate censored events in each group. PCA, principal component analysis; PC1/2, principal component 1/2; IR, immuno relation; Hi, high; Lo, low; CT, center of the tumor; IM, invasive margin; HLA, human leukocyte antigen.

were considered not determined and inserted into the matrix of a table. All RDI values of repertoires were described in the upper right columns and rows. The RDI values showing significant similarities based on both Fisher's exact tests and Mann Whitney U tests were described in the lower left columns and rows.

Other statistical analyses. Clinicopathological variables and IHC data were analyzed using JMP v13.0. software. The associations between the clinicopathological factors and IR groups were assessed using unpaired Student's t-test, $\chi^{2}$ tests and the Fisher's exact tests. Postoperative recurrence-free survival (RFS) and cancer-specific survival (CSS) rates were calculated using the Kaplan-Meier method, and differences in survival between groups were compared using the log-rank test. Comparisons of each T-cell subset marker CD274 and HLA were performed using the Kruskal-Wallis test followed by Dunn's test. $\mathrm{P}<0.05$ was considered to indicate a statistically significant difference.

\section{Results}

Identification of IR group and clinicopathological analyses. In total, 53 patients with surgically treated ESCC were enrolled in the present study. IHC staining analysis with T-cell subset markers and relevant immune checkpoint molecule markers was performed. As shown in Fig. S4, the expression of each T-cell marker was significantly increased in IM than in CT. Additionally, the expression levels of each marker were significantly correlated between the IM and CT via linear regression analysis (Fig. S5).

The 53 patients in the present study were divided into two clusters according to the PCA. As shown in Fig. 1A, the target cases were classified into Cluster_1 $(n=32)$ and Cluster_2 $(n=21)$ and the utilized markers were all concentrated in Cluster_2. Thus, Cluster_1 and Cluster_2 were defined as the IR-Lo and IR-Hi groups, respectively.

The impact of the immune involvement on the prognosis of patients was assessed by comparing results between the IR-Hi and IR-Lo groups. CSS and RFS rates were significantly improved in the IR-Hi group compared with in the IR-Lo group (Fig. 1B and C). Clinicopathological variables were compared between the IR-Hi and IR-Lo groups to reveal the immune involvement and clinical features. There were no significant differences in patients' age, sex, tumor depth, lymphatic and vascular invasion, tumor infiltrating pattern, tumor grading, pathological stage and pathological prognostic group; however, lymph node metastasis was relatively higher in the IR-Lo group than in the IR-Hi group (Table I). 
Table I. Comparisons of clinicopathological variables between the IR-Hi $(n=21)$ and IR-Lo ( $\mathrm{n}=32)$ groups in 53 patients with esophageal squamous cell carcinoma.

\begin{tabular}{|c|c|c|c|}
\hline Clinicopathological variable & IR-Hi (n=21) & IR-Lo $(n=32)$ & P-value \\
\hline Age $\pm \mathrm{SD}$, years & $69.33 \pm 6.59$ & $69.44 \pm 7.13$ & $0.9575^{\mathrm{a}}$ \\
\hline Sex, male/female & $17 / 4$ & $27 / 5$ & $>0.9990^{c}$ \\
\hline Tumor depth, n (\%) & & & $0.6871^{\mathrm{b}}$ \\
\hline $\mathrm{T} 1-\mathrm{T} 2$ & $7(33.33)$ & $9(28.13)$ & \\
\hline T3-T4 & $14(66.67)$ & $23(71.88)$ & \\
\hline Lymphatic invasion, n (\%) & & & $0.0179^{\mathrm{b}}$ \\
\hline ly- & $12(63.16)$ & $7(36.84)$ & \\
\hline ly+ & $9(36.84)$ & $25(73.53)$ & \\
\hline Vascular invasion, $\mathrm{n}(\%)$ & & & $0.4561^{\mathrm{c}}$ \\
\hline $\mathrm{V}-$ & $5(23.81)$ & $4(12.50)$ & \\
\hline $\mathrm{v}+$ & $16(76.19)$ & $28(87.50)$ & \\
\hline INF isoform, $\mathrm{n}(\%)$ & & & $0.4561^{\mathrm{c}}$ \\
\hline$\alpha$ & $5(23.81)$ & $4(12.50)$ & \\
\hline$\beta / \gamma$ & $16(76.19)$ & $28(87.50)$ & \\
\hline Lymph node metastasis, n (\%) & & & $0.1827^{\mathrm{b}}$ \\
\hline N0-N1 & $15(71.43)$ & $17(53.13)$ & \\
\hline N2-N3 & $6(28.58)$ & $15(46.88)$ & \\
\hline Grading, n (\%) & & & $0.1731^{\mathrm{b}}$ \\
\hline G1 & $9(42.86)$ & $8(25.00)$ & \\
\hline G2-G3 & $12(57.14)$ & $24(75.00)$ & \\
\hline TNM pathological stage, n (\%) & & & $0.2305^{\mathrm{b}}$ \\
\hline Stage I-II & $10(47.62)$ & $10(31.25)$ & \\
\hline Stage III-IV & $11(52.38)$ & $22(68.75)$ & \\
\hline Pathological prognostic group, n (\%) & & & $0.2305^{b}$ \\
\hline Group I-II & $10(47.62)$ & $10(31.25)$ & \\
\hline Group III-IV & $11(52.38)$ & $22(68.75)$ & \\
\hline
\end{tabular}

TNM stage and the prognostic group were determined according to the TNM Classification of Malignant Tumors 8th Edition. INF, pattern of tumor infiltration; $\alpha$, expanding growth and a distinct border with the surrounding tissue; $\beta$, in-between $\beta$ and $\gamma ; \gamma$, infiltrating growth and an indistinct border with surrounding tissue; G1, well differentiated; G2, moderately differentiated; G3, poorly differentiated; IR, immuno

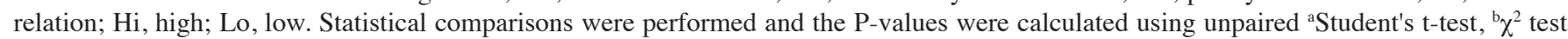
and 'Fisher's exact test.

TCR repertoire analysis. Initially, five patients were randomly selected from the IR-Hi group. Subsequently, the five corresponding patients with matched backgrounds were chosen from the IR-Lo group for comprehensive TCR repertoire analysis using unbiased PCR amplification and NGS analysis of TCR genes. The pathological findings of the 10 ESCC cases are shown in Table II, and images of H\&E-stained and CD3 IHC tumor tissue samples are presented in Fig. S6.

In the comprehensive analysis of the TCR repertoire, $1,671,742$ and 1,291,510 total reads of the TCR $\alpha$ and TCR $\beta$ sequences, respectively, were obtained from the 10 cases; of these reads, 31,954 and 34,876 were unique, respectively (Table SIII). In cases 1 and 3 in the IR-Hi group, a single VJ region recombination amplification was observed for TCR $\alpha$, and the diversity of the TCR $\alpha$ repertoire seemed to be lost. In case 1, 34,671 reads were obtained for recombination of TRAV19|TRAJ41 regions. In case 3, 24,315 reads were obtained for recombination of TRAV39ITRAJ58 regions. No other cases showed a single recombinant repertoire amplification (Fig. 2). In terms of the IR-Lo group, case 9 showed a single VJ recombination amplification (TRAV19ITRAJ12) and the reduction of its diversity in the TCR $\alpha$ repertoire. There seemed to be no marked differences in TCR diversity between IR-Hi and IR-Lo groups (Figs. 2 and S7). The subsequent investigation was performed to compare the TCR repertoire differences between the IR-Hi and the IR-Lo groups.

TCR repertoire dissimilarity analysis and shared TCR VJ region. The RDI of the TCR was investigated to determine whether there were diversities in the VJ regions in the IR-Hi group. Among the 10 cases in which the indexes were investigated, RDIs were conserved in the IR-Hi group; however, the index could not be calculated in the IR-Lo group owing to the lack of sufficient numbers in the repertoire. In TCR $\alpha$ 


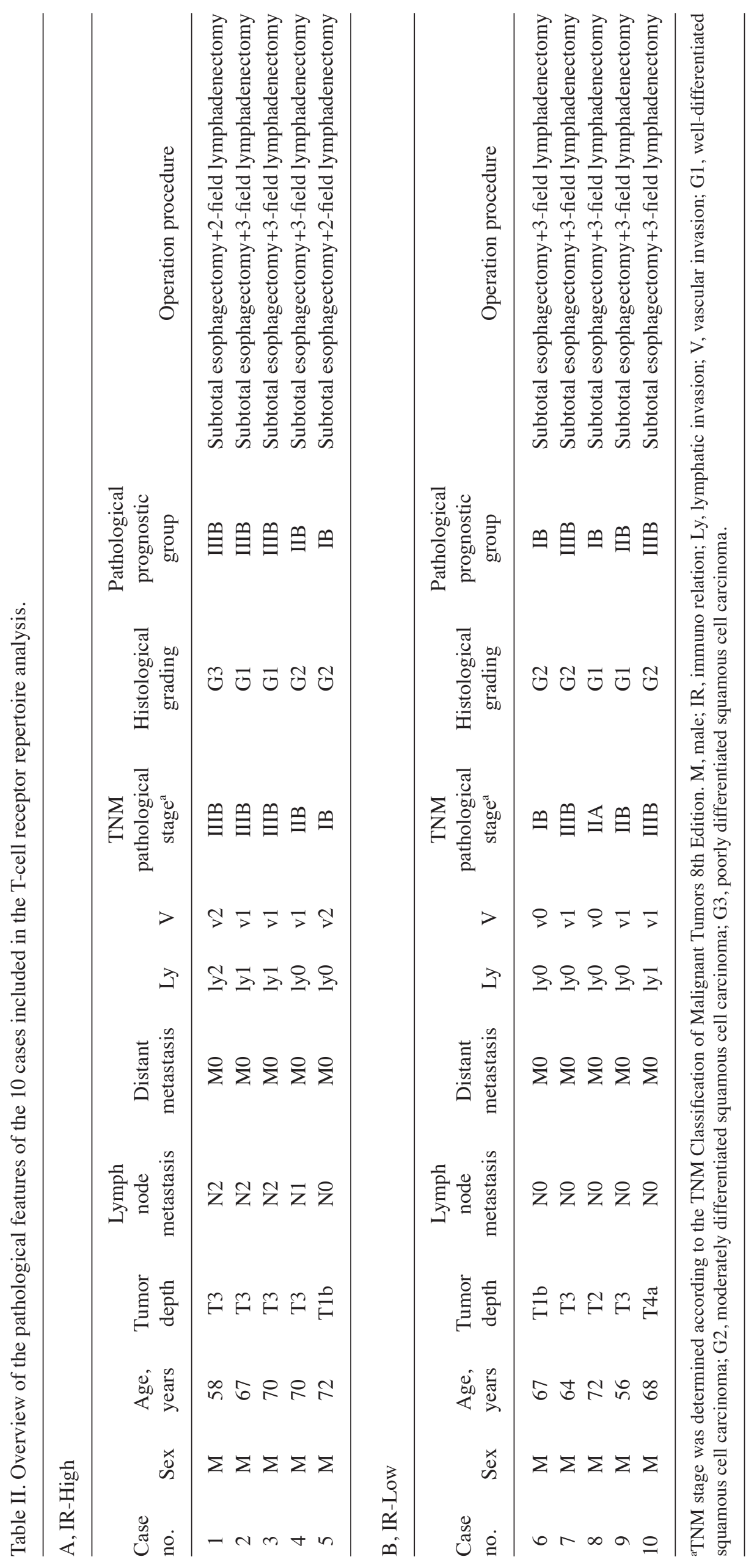


$\operatorname{TCR} \alpha$
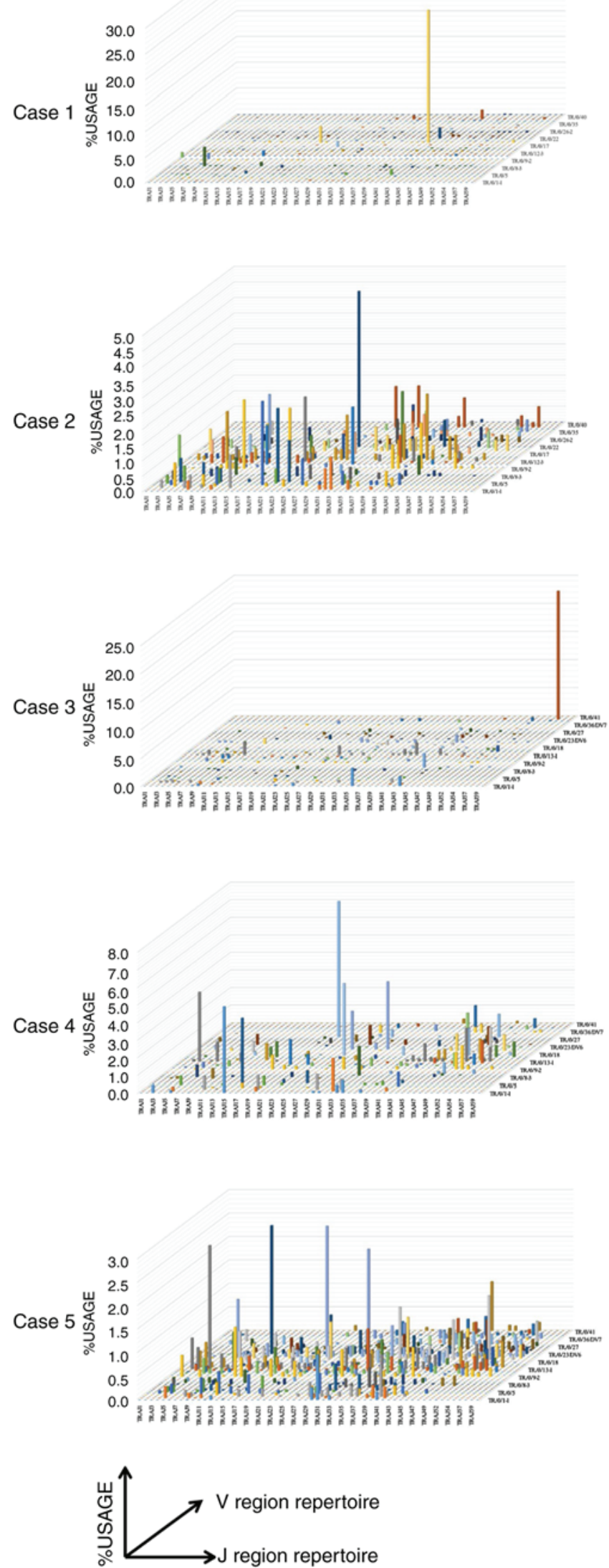

TCR $\beta$
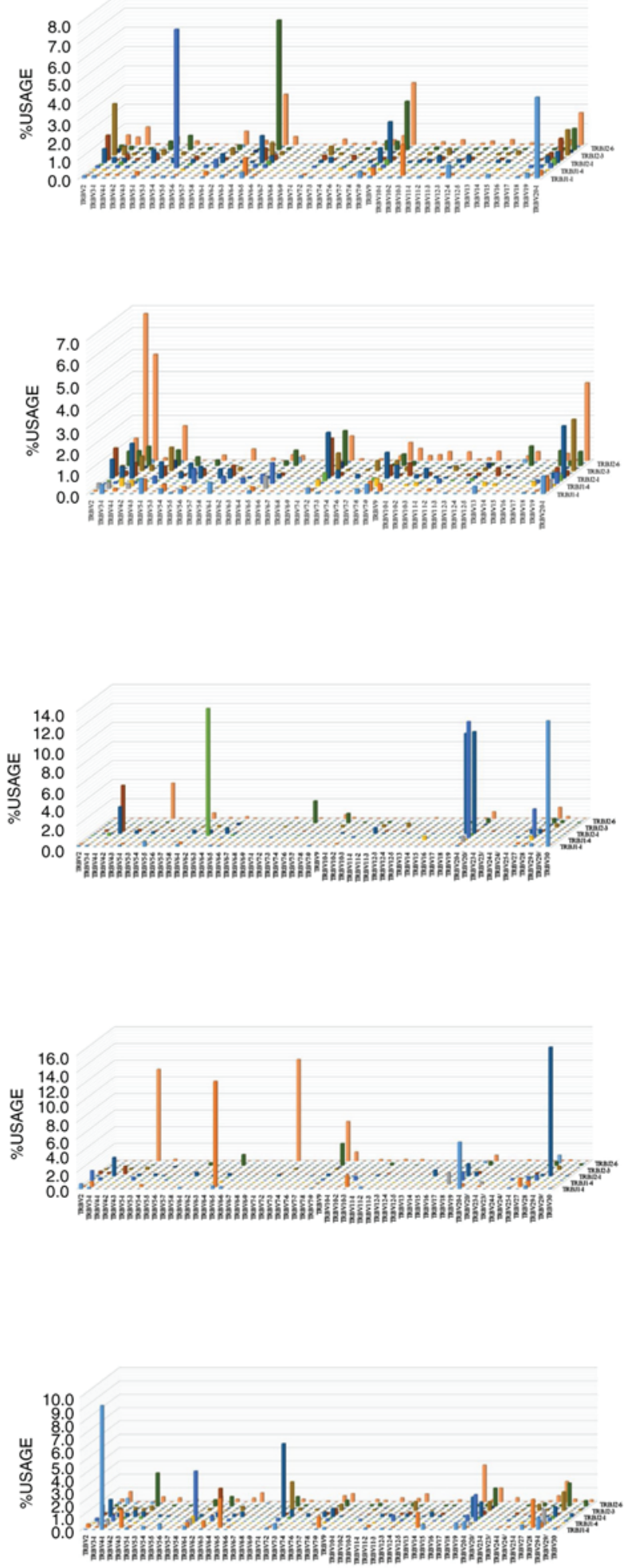

Figure 2. Three-dimensional graphic images of the TCR overview of the diversities of the T-cell repertoires among five immuno relation-high cases. The X-axis represents the repertoire of the joining regions, and the $y$-axis represents the repertoire of the variable regions. The height of each bar indicates the readout frequency of the combination of TCR $\alpha \mathrm{V}$ and J regions, and TCR $\beta \mathrm{V}$ and J regions by next-generation sequencing analysis. TCR $\alpha$ and TCR $\beta$ repertoires are shown in the left and the right columns, respectively. TCR, T-cell receptor; V, variable; J, joining; TRAV, T-cell receptor $\alpha$ variable region; TRAJ, T-cell receptor $\alpha$ joining region. 


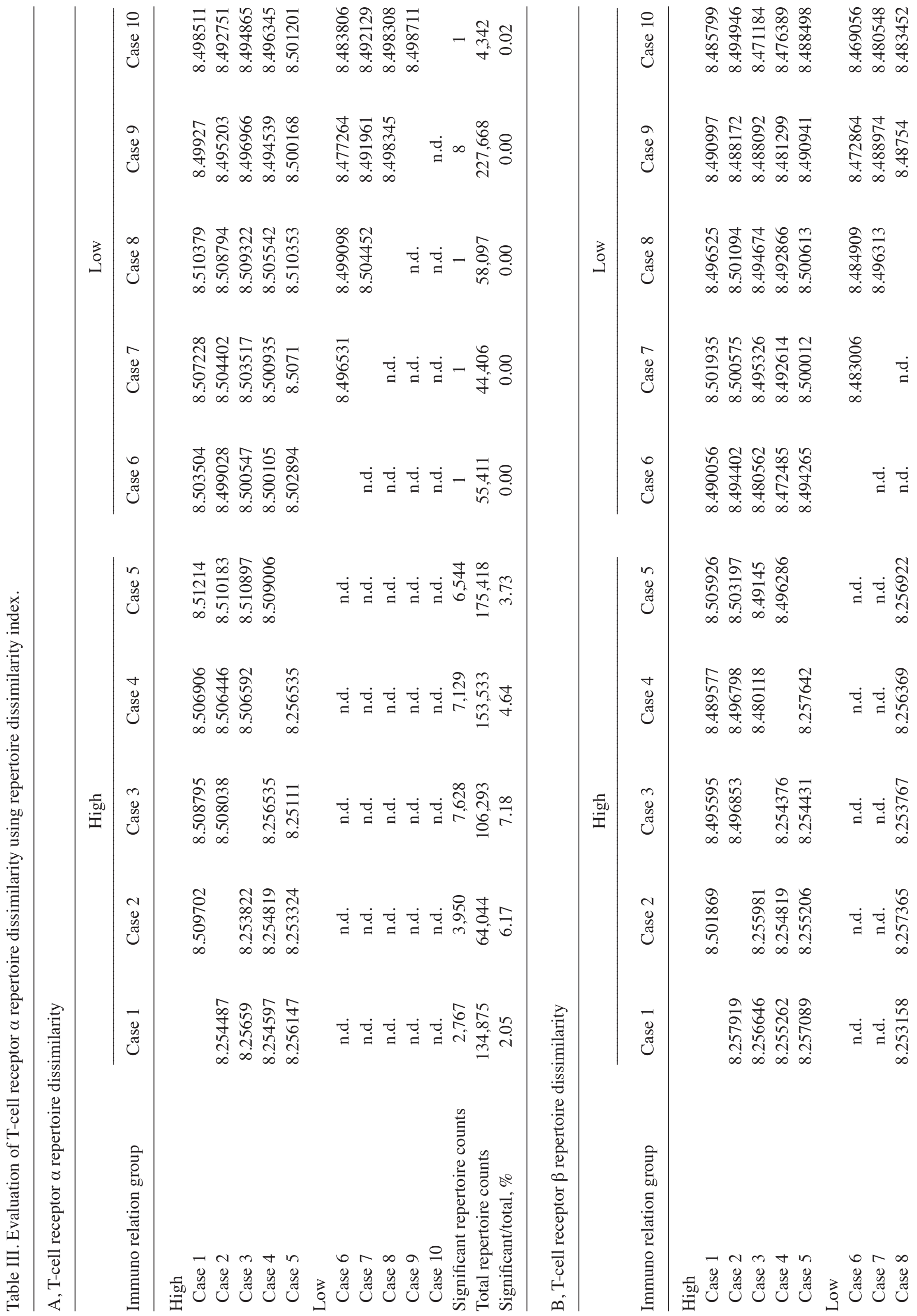


and TCR $\beta$, the rates of significant diverse repertoires were 2-7 and $6-11 \%$, respectively, in the IR-Hi group; by contrast, there were no diverse repertoires other than for case 8 for the TCR $\beta$ repertoire in the IR-Lo group (Table III). The total repertoire TCR counts of TCR $\alpha$ and TCR $\beta$ did not differ significantly (Fig. 3A and B); however, the significant repertoire TCR counts were significantly higher in the IR-Hi group compared with in the IR-Lo group (Fig. 3C and D).

Among the significantly diverse TCR $\alpha$ and TCR $\beta$ repertoire, the shared recombinant sequences in the $\mathrm{VJ}$ region were explored. Fisher's exact tests were used, followed by Mann Whitney U tests, to ensure the significance of the results. In total, 27 TRAVITRAJ combinations for TCR $\alpha$ and 23 TRBVITRBJ combinations for TCR $\beta$ were identified as having shared repertoires (Table IV). In particular, in the IR-Hi group, the read-out numbers for the combinations of TRAV13-1ITRAJ44 and TRAV13-1ITRAJ22 for TCR $\alpha$ and the combinations of TRBV7-9|TRBJ2-7 and TRBV20-1ITRBJ1-1 for TCR $\beta$ were consistently high.

T-cell subset cluster analysis in the IR-Hi group. Exploratory visualization utilizing hierarchical clustering analysis was then performed to assess whether the T-cell subset imbalance had any influence in the IR-Hi group. The IR-Hi group was classified in three clusters (Fig. 4A), namely Cluster_1 ( $n=3)$, Cluster_2 $(\mathrm{n}=15)$ and Cluster_3 $(\mathrm{n}=3)$.

Next, each T-cell subset marker, as well as CD274 and HLA, were compared to determine whether the imbalances existed within the three clusters. The expression levels of CD8 and FOXP3 were significantly higher in Cluster_1 and Cluster 3, respectively, compared with in Cluster_2 (Fig. 4B and D). However, there were no significant differences with regard to CD45RO, CD274 and HLA among the three clusters (Fig. 4C, E and F). The CSS and RFS for each cluster were compared, and there were no significant differences among the 3 clusters; however, cluster 1 tended to have a less favourable RFS outcome than the other clusters (Fig. S8).

\section{Discussion}

The present study indicated that the prognosis of patients with ESCC could be stratified according to IR status, which was evaluated based on T-cell subset markers CD274 and HLA. A comprehensive TCR analysis and comparison between the IR-Hi and IR-Lo groups indicated that the IR-Hi group exhibited a diverse TCR repertoire and shared $\mathrm{V} / \mathrm{J}$ regions recombination in TCR $\alpha$ and $\beta$ chains. In the IR-Hi group, the prognosis of the patients could be further stratified into three clusters according to the expression patterns of $\mathrm{CD} 8, \mathrm{CD} 45 \mathrm{RO}$ and FOXP3.

The TCR diversity was well conserved and the values of RDI were significantly higher in the IR-Hi group. Thus, the IR-Hi group persistently exhibited the capacity to respond to diverse antigens. TCR diversity is an important element used to evaluate the immunogenicity of tumors and host immune capabilities. The level of the TCR diversity increased as the immunogenicity of the tumor increased. Notably, Carreno et al (33) reported that dendritic vaccination for translation of tumor antigen information into the host immune system increased the TCR diversity. Manuel et al (34) 

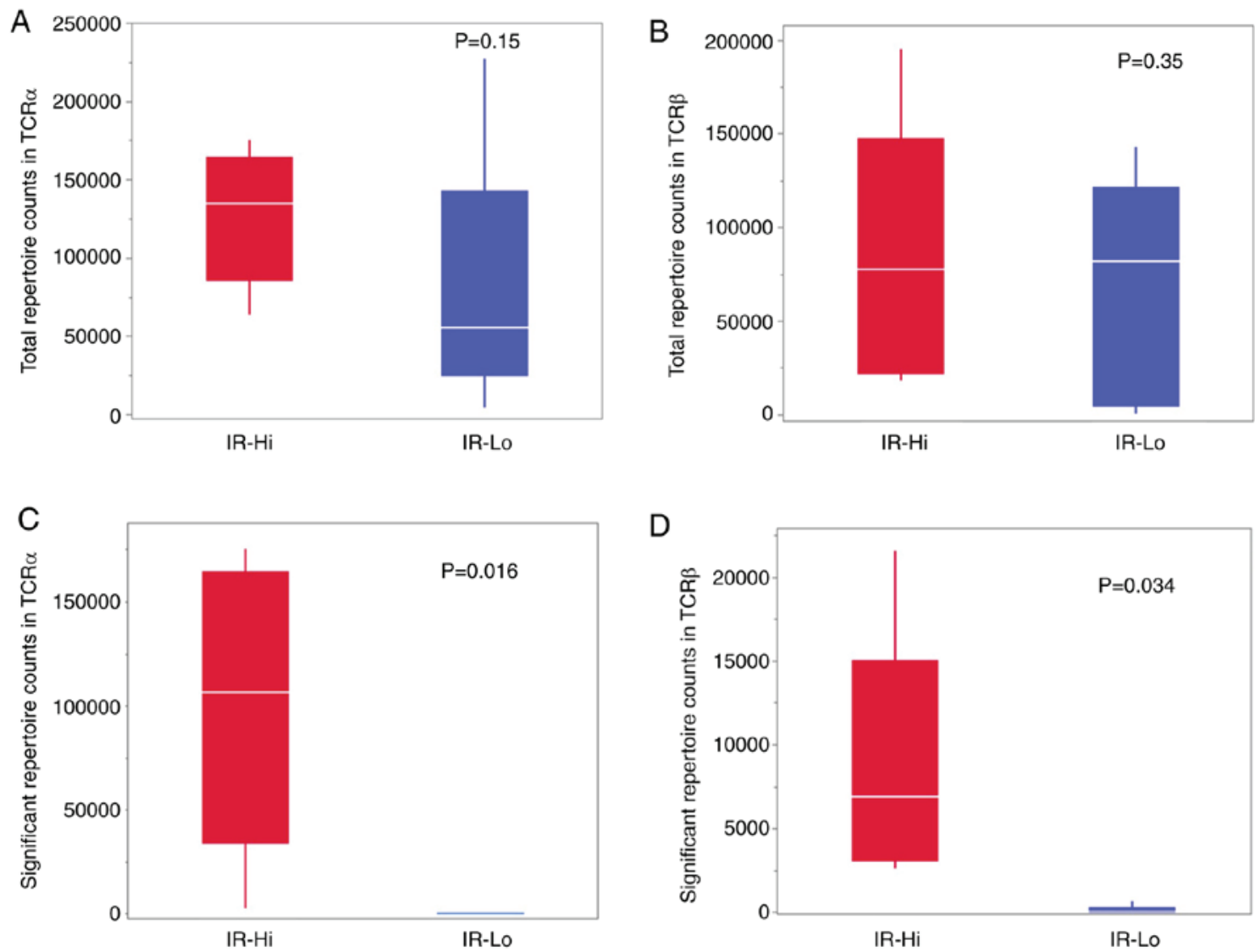

Figure 3. Statistical comparison of TCR repertoires in TCR $\alpha$ and TCR $\beta$ between IR-Hi and IR-Lo groups. Total counts of (A) TCR $\alpha$ repertoire and (B) TCR $\beta$ repertoire. Significant repertoire counts of (C) TCR $\alpha$ and (D) TCR $\beta$. The values were statistically compared with unpaired Student's t-tests. TCR, T-cell receptor; IR, immuno relation; Hi, high; Lo, low.

suggested that the prognosis of patients with metastatic breast cancer was worse in patients with decreasing TCR diversity. Accordingly, the IR-Hi group may be a receptive candidate for immunotherapy since it is in a preferable immune responsive status and holds diverse TCR expression T cells that can respond to various antigens.

In addition to TCR diversity analysis, the present study observed shared $\mathrm{V} / \mathrm{J}$ region recombinations in TCR $\alpha$ and TCR $\beta$. Tan et al (35) screened tumor-reactive TILs specifically to recognize fragments from autologous tumor cells and identified nine TRAV/TRAJ and four TRBV/TRBJ recombination sequences via TCR analysis. Notably, TRAV13-1/TRAJ22 and TRBV7-9/TRBJ2-7 are both candidates identified from the aforementioned study (35), and the same combinations were identified in the present study. Additionally, Tan et al (35) provided evidence that these TCRs could work for tumor-reactive; however, their study was conducted using the sample acquired from one patient. If there is a tumor-reactive $\mathrm{T}$ cell with the shared TCR in patients with ESCC, it implies the existence of an antigen that holds the common portion for the $\mathrm{T}$ cell reactivity. Thus, the shared TCR repertoires in the IR-Hi group that were identified in the present study may be important for investigating the novel antigen.

In the hierarchical cluster analysis, the IR-Hi group was divided into three clusters according to the expression patterns of CD8, CD45RO and FOXP3. There were no significant differences in prognosis among the clusters; however, the RFS rate in Cluster_3 was the highest and that in Cluster_1 the least favourable. When considering T-cell subset functions, the RFS of Cluster_1, which showed abundant cytotoxic and memory T cells, was expected to be better than that of Cluster_3, which was rich in regulatory $\mathrm{T}$ cells. However, the result was contradictory to the expectation. With regards to these findings, six immune subtype clusters ( $\mathrm{C} 1$, wound healing; $\mathrm{C} 2, \mathrm{IFN}-\gamma$ dominant; $\mathrm{C} 3$, inflammatory; $\mathrm{C} 4$, lymphocyte depleted; $\mathrm{C}$, immunologically quiet; and C6, TGF- $\beta$ dominant subtypes) were proposed through the gene expression profile of TILs using The Cancer Genome Atlas data (36). According to the report, the $\mathrm{C} 2$ subtype with a hyper cytotoxic T-cell signature had a poor prognosis than that of the C3 subtype with a balanced macrophage and $\mathrm{T}$ cell signature. As suggested by Thorsson et al (36), exacerbating the cytotoxic dominant condition in the tumor microenvironment may favor tumor growth, whereas a well-balanced T-cell subset between cytotoxic and regulatory signatures may be necessary for optimal immunological antitumor function.

Considering the IR-status and hierarchical clustering in a clinical setting, the IR-Lo group would not be indicated for immunotherapy since its immune profile would be low, so conventional chemoradiotherapy or surgery may be preferable. On the contrary, in the IR-Hi group, Cluster_3 would be the most preferable group for immunotherapy since its immune profile is high and the T-cell subset is well balanced. Additionally, comprehensive TCR repertoire analysis may 


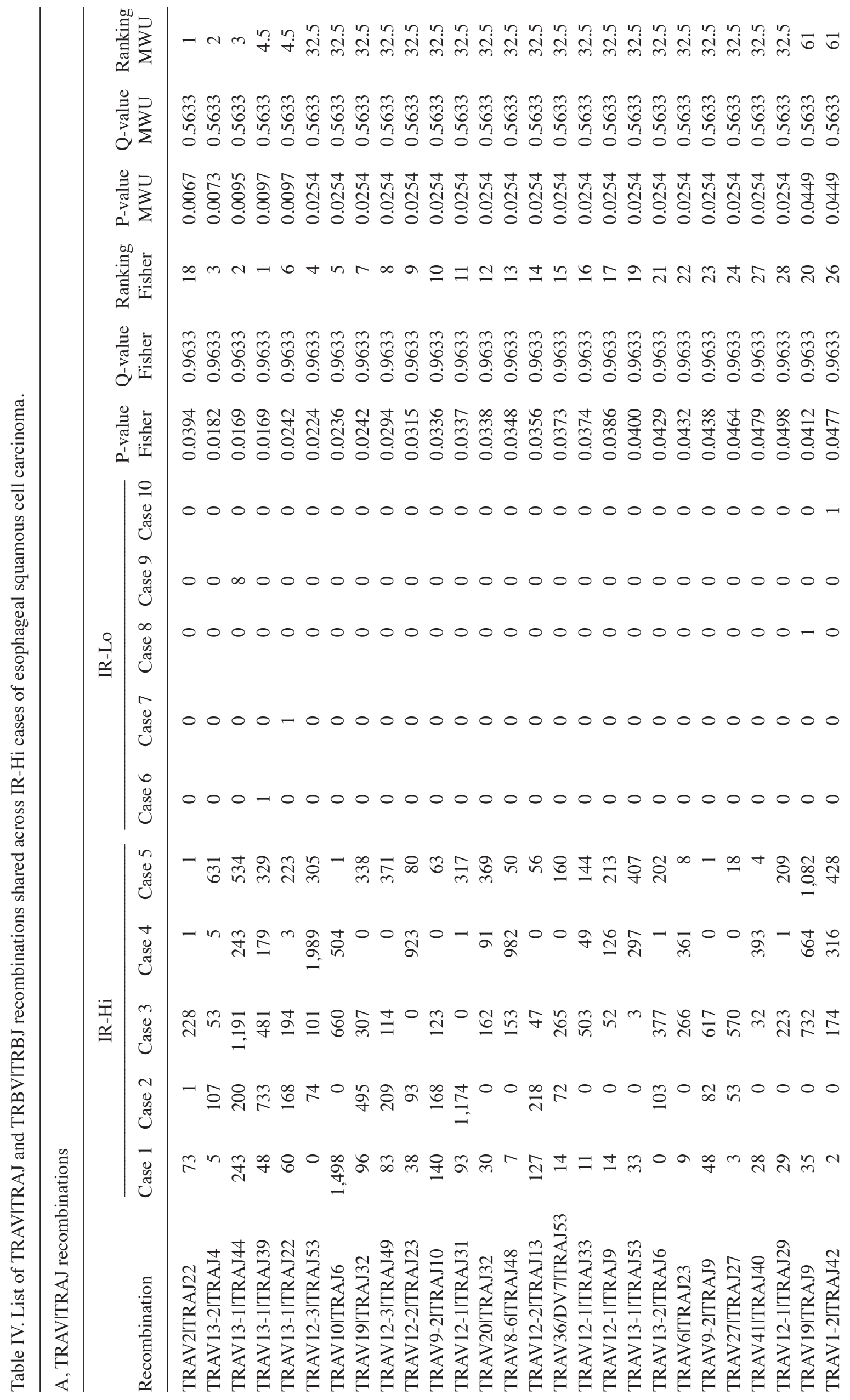




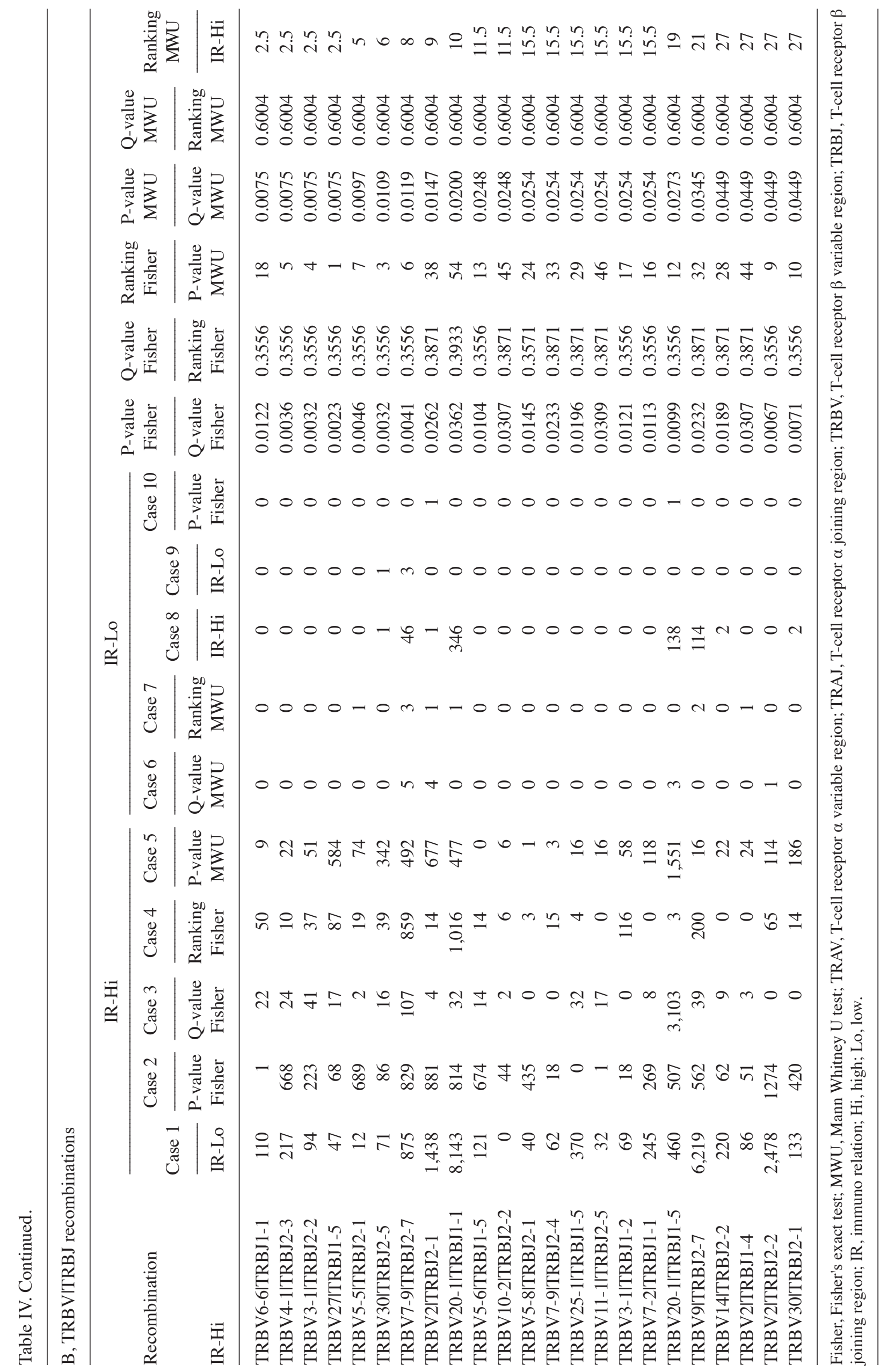


A

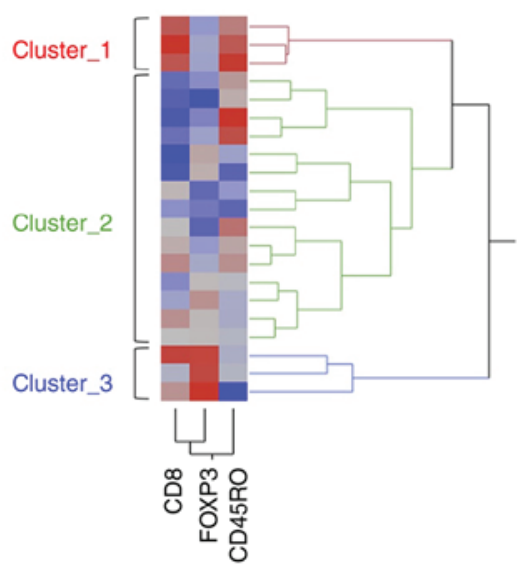

B

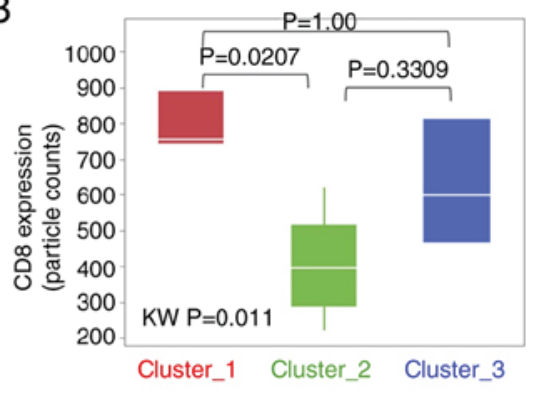

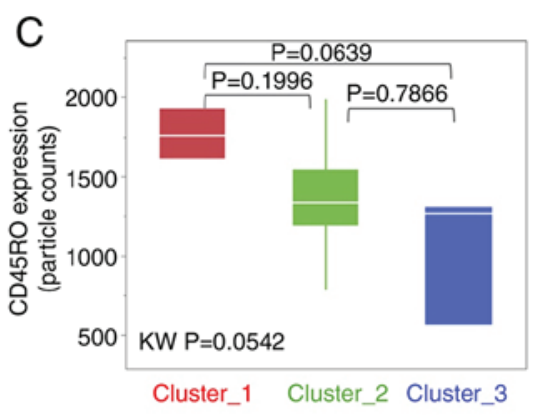

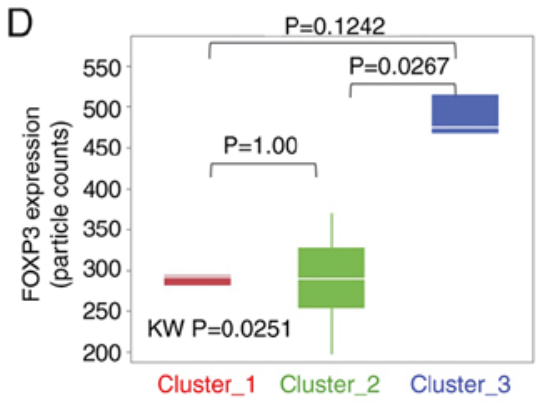

E

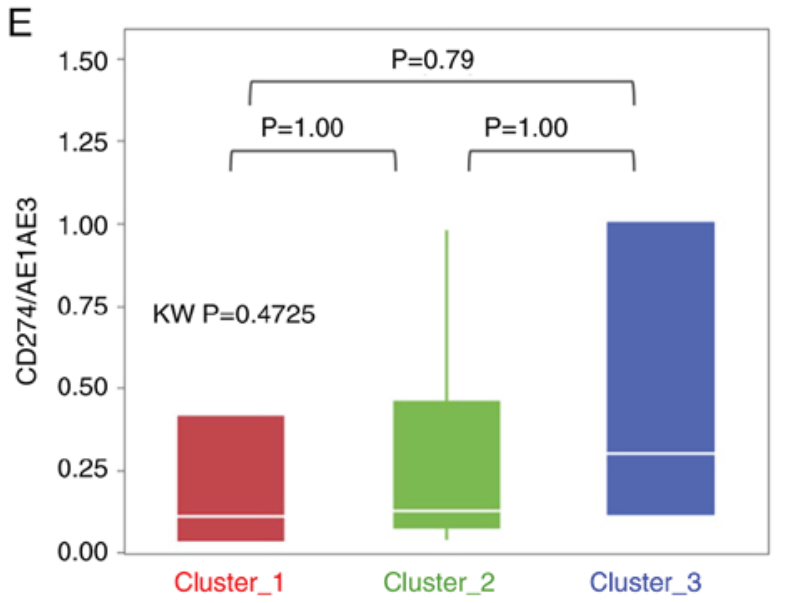

$\mathrm{F}$

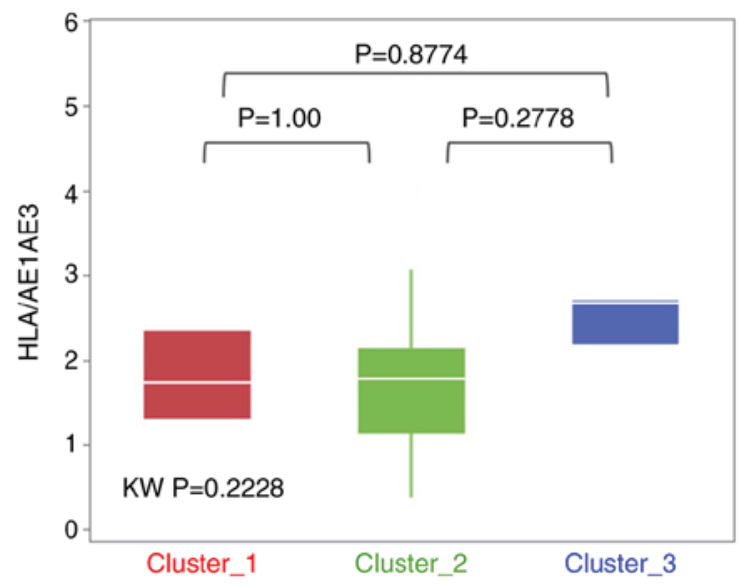

Figure 4. Clustering analysis in the immuno relation-high group and comparison of T-cell subset markers and CD274 and HLA expression in each cluster. (A) Hierarchical clustering analysis using Ward's method. Red and blue plots indicate positive and negative correlation, respectively. The expression of each marker was compared using the KW test followed by Dunn's test among the three clusters. Comparison of expression levels of (B) CD8, (C) CD45RO, (D) FOXP3, (E) CD274 and (F) HLA. The y-axes indicate the expression values calculated by ImageJ software. KW, Kruskal-Walis; HLA, human leukocyte antigen.

facilitate the discovery of novel common cancer antigens and the development of CAR-T cell therapy in ESCC.

In the present study, the $\mathrm{V} / \mathrm{J}$ recombination sequences were narrowed down using two different statistical procedures and several combinations were commonly shared in the IR-Hi group. By combining multiple statistical methods, the present study attempted to ascertain the reliability of the $\mathrm{V} / \mathrm{J}$ recombination candidate. Notably, the selected candidates contained the recombination sequences that were involved in $\mathrm{T}$ cell activation and cytokine release (35), thus the results presented here are reasonable.

Adaptor ligation PCR and NGS analysis were performed utilizing the mRNA samples collected from bulk tumor tissues in the present study. There may be concerns for the credibility of the TCR analysis without using laser micro dissection or other equivalent techniques; however, the procedures performed in the present study have been previously reported in several studies (37-39), and the reliability of the technique is expected to be sufficient. Moreover, as shown in Fig. S4, T cells were spatially accumulated surrounding the tumor, therefore bulk samples containing surrounding tumor structures may be appropriate for obtaining TCR information.

However, there are some limitations in the present study. The study was conducted with a relatively small number of cases, which may have limited the conclusions of the findings. The TCR repertoire analysis, which suggested the existence of shared $\mathrm{V} / \mathrm{J}$ region recombinations and corresponding antigens, included only a few cases, and additional CDR3 clonotypes investigation should be conducted. Additionally, the TCR $\alpha$ and $\beta$ chains were investigated separately. T cells recognize antigens via heterodimerization of the TCR $\alpha$ and TCR $\beta$ chains, thus it was not clear which antigen was recognized 
by the common $\mathrm{V} / \mathrm{J}$ recombination regions confirmed in the ESCC cases. Furthermore, the variations in TCR $\alpha \beta$ repertoire combination were large, and it may be possible that candidates were selected by chance. Therefore, further investigations are warranted in future studies.

In conclusion, the results of the present study confirmed two distinctive subgroups in ESCC according to the T cell subset marker expression. Furthermore, abundant TCR repertoire diversity partly containing shared $\mathrm{V} / \mathrm{J}$ region recombination in the IR-Hi group was confirmed, which may allow development of a novel immune-oriented therapy.

\section{Acknowledgements}

The authors would like to thank Ms. Matsuo from the Department of Surgery, Kurume University (Kurume, Japan) and Ms. Kawaguchi from Research for Innovative Cancer Therapy, Kurume University (Kurume, Japan) for performing the RNA extractions from the tissue samples, and Ms. Otsu fom the Department of Surgery, Kurume University (Kurume, Japan) for the preparation of the slides for H\&E and IHC staining.

\section{Funding}

The present study was supported by a Grant-in-Aid for Scientific Research (grant no. 16K07130).

\section{Availability of data and materials}

The datasets generated and/or analyzed during the current study are available in the DDBJ BioProject repository (http://trace. ddbj.nig.ac.jp/BPSearch/bioproject?acc=PRJDB9359) and the NCBI repository (https://www.ncbi.nlm.nih. gov/bioproject/?term=PRJDB9359).

\section{Authors' contributions}

TS planned all experiments and wrote the article. KI performed the computational analysis. AK and JA performed immunohistochemical staining and pathological diagnosis. SN, MN, HK, MF, $\mathrm{HH}, \mathrm{KS}, \mathrm{SM}$ and NM collected and interpreted the clinical data for the enrolled patients. TS and KI confirmed the authenticity of all raw data. AM helped to perform the final pathological diagnosis and provided a critical suggestion about the interpretation of the IHC staining materials. AY helped to interpret the $\mathrm{T}$ cell receptor repertoire analysis. AM and AY were involved in drafting the initial manuscript and critically revising it for important intellectual content. YA supervised the research group, conceived the study, helped to write the discussion section and gave final approval of the version to be published. All authors have read and approved the final manuscript.

\section{Ethics approval and consent to participate}

The present study was conducted under the provision of the Declaration of Helsinki and was approved by the Institutional Review Board of Kurume University Hospital (Kurume, Japan; approval no. 282). Written informed consent was obtained from all enrolled patients.

\section{Patient consent for publication}

Not applicable.

\section{Competing interests}

The authors declare that they have no competing interests.

\section{References}

1. Bray F, Ferlay J, Soerjomataram I, Siegel RL, Torre LA and Jemal A: Global cancer statistics 2018: GLOBOCAN estimates of incidence and mortality worldwide for 36 cancers in 185 countries. CA Cancer J Clin 68: 394-424, 2018.

2. Domper Arnal MJ, Ferrández Arenas Á and Lanas Arbeloa Á: Esophageal cancer: Risk factors, screening and endoscopic treatment in Western and Eastern countries. World J Gastroenterol 21: 7933-7943, 2015.

3. Ide H, Nakamura T, Hayashi K, Endo T, Kobayashi A, Eguchi R and Hanyu F: Esophageal squamous cell carcinoma: Pathology and prognosis. World J Surg 18: 321-330, 1994.

4. Matsuda S, Takeuchi H, Kawakubo H, Ando N and Kitagawa Y: Current advancement in multidisciplinary treatment for resectable cStage II/III esophageal squamous cell carcinoma in Japan. Ann Thorac Cardiovasc Surg 22: 275-283, 2016.

5. Watanabe M, Otake R, Kozuki R, Toihata T, Takahashi K, Okamura A and Imamura Y: Correction to: Recent progress in multidisciplinary treatment for patients with esophageal cancer. Surg Today 50: 425, 2020.

6. Shah MA, Kojima T, Hochhauser D, Enzinger P, Raimbourg J, Hollebecque A, Lordick F, Kim SB, Tajika M, Kim HT, et al: Efficacy and safety of pembrolizumab for heavily pretreated patients with advanced, metastatic adenocarcinoma or squamous cell carcinoma of the esophagus: The phase 2 KEYNOTE-180 study. JAMA Oncol 5: 546-550, 2019.

7. Kojima T, Shah MA, Muro K, Francois E, Adenis A, Hsu CH, Doi T, Moriwaki T, Kim SB, Lee SH, et al: Randomized phase III KEYNOTE-181 study of pembrolizumab versus chemotherapy in advanced esophageal cancer. J Clin Oncol 38: 4138-4148, 2020.

8. Sudo T, Nishida R, Kawahara A, Saisho K, Mimori K, Yamada A, Mizoguchi A, Kadoya K, Matono S, Mori N, et al: Clinical impact of tumor-infiltrating lymphocytes in esophageal squamous cell carcinoma. Ann Surg Oncol 24: 3763-3770, 2017.

9. Ohtani H: Focus on TILs: Prognostic significance of tumor infiltrating lymphocytes in human colorectal cancer. Cancer Immun 7: 4, 2007.

10. Huh JW, Lee JH and Kim HR: Prognostic significance of tumor-infiltrating lymphocytes for patients with colorectal cancer. Arch Surg 147: 366-372, 2012.

11. Kang WB, Seo NA, Yoon S, Bae HI, Jeon SW, Kwon OK, Chung HY, Yu W, Kang $\mathrm{H}$ and Kim JG: Prognostic value of tumor-infiltrating lymphocytes in Epstein-Barr virus-associated gastric cancer. Ann Oncol 27: 494-501, 2016.

12. Zhang D, He W, Wu C, Tan Y, He Y, Xu B, Chen L, Li Q and Jiang J: Scoring system for tumor-infiltrating lymphocytes and its prognostic value for gastric cancer. Front Immunol 10: 71, 2019.

13. Lu G, Chen L, Wu S, Feng Y and Lin T: Comprehensive analysis of tumor-infiltrating immune cells and relevant therapeutic strategy in esophageal cancer. Dis Markers 2020: 8974793, 2020.

14. Giannakis M, Mu JX, Shukla AS, Qian ZR, Cohen O, Nishihara R, Bahl S, Cao Y, Amin-Mansour A, Yamauchi M, et al: Genomic correlates of immune-cell infiltrates in colorectal carcinoma. Cell Rep 15: 857-865, 2016.

15. Tahara H, Shiozaki H, Kobayashi K, Yano T, Yano H, Tamura S, Oku K, Miyata M, Wakasa K, Sakurai M, et al: Phenotypic characteristics of tumour-infiltrating lymphocytes in human oesophageal cancer tissues defined by quantitative two-colour analysis with flow-cytometry. Virchows Arch A Pathol Anat Histopathol 416: 329-334, 1990.

16. Woo EY, Yeh H, Chu CS, Schlienger K, Carroll RG, Riley JL, Kaiser LR and June CH: Cutting edge: Regulatory T cells from lung cancer patients directly inhibit autologous $\mathrm{T}$ cell proliferation. J Immunol 168: 4272-4276, 2002.

17. Ostrand-Rosenberg S: Immune surveillance: A balance between protumor and antitumor immunity. Curr Opin Genet Dev 18: $11-18,2008$. 
18. Heilig SJ and Tonegawa S: Diversity of murine gamma genes and expression in fetal and adult T lymphocytes. Nature 322: 836-840, 1986

19. Postow MA, Manuel M, Wong P, Yuan J, Dong Z, Liu C, Perez S, Tanneau I, Noel M, Courtier A, et al: Peripheral T cel receptor diversity is associated with clinical outcomes following ipilimumab treatment in metastatic melanoma. J Immunother Cancer 3: 23, 2015.

20. Cha E, Klinger M, Hou Y, Cummings C, Ribas A, Faham M and Fong L: Improved survival with $\mathrm{T}$ cell clonotype stability after anti-CTLA-4 treatment in cancer patients. Sci Transl Med 6: 238ra70, 2014.

21. Robert L, Tsoi J, Wang X, Emerson R, Homet B, Chodon T, Mok S, Huang RR, Cochran AJ, Comin-Anduix B, et al: CTLA4 blockade broadens the peripheral T-cell receptor repertoire. Clin Cancer Res 20: 2424-2432, 2014.

22. Li C, Zhang Y, Zhang C, Chen J, Lou X, Chen X, Kang L, Xu N, Li M, Tan J, et al: Comparation of CART19 and autologous stem-cell transplantation for refractory/relapsed non-Hodgkin's lymphoma. JCI Insight 5: e130195, 2019.

23. Hu K, Kang N, Liu Y, Guo D, Jing W, Lu J, Tan T, Lv C, Deng Y, Long J, et al: Proposed revision of $\mathrm{N}$ categories to the 8th edition of the AJCC-TNM staging system for non-surgical esophageal squamous cell cancer. Cancer Sci 110: 717-725, 2019.

24. Schneider AC, Rasband SW and Eliceiri WK: NIH image to imageJ: 25 years of image analysis. Nat Methods 9: 671-675, 2012

25. Yamaguchi-Kabata Y, Nakazono K, Takahashi A, Saito S, Hosono N, Kubo M, Nakamura Y and Kamatani N: Japanese population structure, based on SNP genotypes from 7003 individuals compared to other ethnic groups: Effects on population-based association studies. Am J Hum Genet 83: 445-456, 2008.

26. Armstrong SA, Staunton JE, Silverman LB, Pieters R, den Boer ML, Minden MD, Sallan SE, Lander ES, Golub TR and Korsmeyer SJ: MLL translocations specify a distinct gene expression profile that distinguishes a unique leukemia. Nat Genet 30: 41-47, 2002.

27. Matsutani T, Yoshioka T, Tsuruta Y, Iwagami S and Suzuki R: Analysis of TCRAV and TCRBV repertoires in healthy individuals by microplate hybridization assay. Hum Immunol 56 57-69, 1997.

28. Kitaura K, Shini T, Matsutani T and Suzuki R: A new high-throughput sequencing method for determining diversity and similarity of T cell receptor (TCR) $\alpha$ and $\beta$ repertoires and identifying potential new invariant TCR $\alpha$ chains. BMC Immunol 17: 38, 2016.

29. McCarthy JD, Chen Y and Smyth KG: Differential expression analysis of multifactor RNA-Seq experiments with respect to biological variation. Nucleic Acids Res 40: 4288-4297, 2012.
30. Robinson DM, McCarthy JD and Smyth KG: EdgeR: A bioconductor package for differential expression analysis of digital gene expression data. Bioinformatics 26: 139-140, 2010.

31. Bolen CR, Rubelt F, Vander Heiden JA and Davis MM: The repertoire dissimilarity index as a method to compare lymphocyte receptor repertoires. BMC Bioinformatics 18: 155, 2017.

32. Rubelt F, Bolen CR, McGuire HM, Vander Heiden JA, Gadala-Maria D, Levin M, Euskirchen GM, Mamedov MR, Swan GE, Dekker CL, et al: Individual heritable differences result in unique cell lymphocyte receptor repertoires of naïve and antigen-experienced cells. Nat Commun 7: 11112, 2016.

33. Carreno MB, Magrini V, Becker-Hapak M, Kaabinejadian S, Hundal J, Petti AA, Ly A, Lie WR, Hildebrand WH, Mardis ER and Linette GP: Cancer immunotherapy. A dendritic cell vaccine increases the breadth and diversity of melanoma neoantigen-specific T cells. Science 348: 803-808, 2015.

34. Manuel M, Tredan O, Bachelot T, Clapisson G, Courtier A, Parmentier G, Rabeony T, Grives A, Perez S, Mouret JF, et al: Lymphopenia combined with low TCR diversity (divpenia) predicts poor overall survival in metastatic breast cancer patients. Oncoimmunology 1: 432-440, 2012.

35. Tan Q, Zhang C, Yang W, Liu Y, Heyilimu P, Feng D, Xing L, $\mathrm{Ke} \mathrm{Y}$ and $\mathrm{Lu} \mathrm{Z}$ : Isolation of $\mathrm{T}$ cell receptor specifically reactive with autologous tumour cells from tumour-infiltrating lymphocytes and construction of $\mathrm{T}$ cell receptor engineered $\mathrm{T}$ cells for esophageal squamous cell carcinoma. J Immunother Cancer 7: 232, 2019.

36. Thorsson V, Gibbs LD, Brown DS, Wolf D, Bortone DS, Ou Yang TH, Porta-Pardo E, Gao GF, Plaisier CL, Eddy JA, et al: The Immune landscape of cancer. Immunity 48: 812-830.e14, 2018.

37. Saitoh H, Hirokawa M, Fujishima N, Ichikawa Y, Kawabata Y, Miura I, Miura AB, Matsutani T, Suzuki R and Sawada K: The presence and longevity of peripherally expanded donor-derived TCRalphabeta+ mature $\mathrm{T}$ lymphocyte clones after allogeneic bone marrow transplantation for adult myeloid leukemias. Leukemia 17: 1626-1635, 2003.

38. Matsutani T, Yoshioka T, Tsuruta Y, Shimamoto T, Ohyashiki JH, Suzuki R and Ohyashiki K: Determination of T-cell receptors of clonal CD8-positive T-cells in myelodysplastic syndrome with erythroid hypoplasia. Leuk Res 27: 305-312, 2003.

39. Matsutani T, Ohashi Y, Yoshioka T, Tsuruta Y, Doi H, Satomi S and Suzuki R: Skew in T-cell receptor usage and clonal T-cell expansion in patients with chronic rejection of transplanted kidneys. Transplantation 75: 398-407, 2003.

This work is licensed under a Creative Commons Attribution-NonCommercial-NoDerivatives 4.0 International (CC BY-NC-ND 4.0) License. 